\title{
Concentración de plomo, mercurio y cadmio en músculo de peces y muestras de agua procedentes del Río Santa, Ancash - Perú
}

\begin{abstract}
Concentration of lead, mercury and cadmium in fish muscle and water samples from the Rio Santa, Ancash
\end{abstract} - Peru

\author{
Flavia Bertolotti Rivera ${ }^{1}$, Norma Noé Moccetti ${ }^{2}$
}

\section{RESUMEN}

El objetivo del estudio fue cuantificar la concentración de $\mathrm{Pb}, \mathrm{Hg}$ y $\mathrm{Cd}$ en músculo de peces destinado a consumo humano y de muestras de agua de río en tres localidades ( $\mathrm{A}=$ Catac, $\mathrm{B}=$ Taricá y $\mathrm{C}=$ Palmira $)$ de la ciudad de Huaraz (Ancash, Perú). Cinco peces y una muestras de agua en cada localidad fueron analizadas mediante la técnica de Absorción Atómica de Flama. La concentración de Pb en músculos de peces fue 1,826, 0,321 y 0,709 mg/Kg para las zonas A, B y C respectivamente. En el mismo orden, la concentración de $\mathrm{Hg}$ fue 0,344, 0,433 y 0,284 mg/Kg y de $\mathrm{Cd}, 0,001,0,0$ y $0,002 \mathrm{mg} / \mathrm{Kg}$ respectivamente. En las tres zonas, las concentraciones de $\mathrm{Pb}$ estuvieron por encima de los niveles permitidos por la Comisión Reguladora Europea. La concentración de $\mathrm{Pb}$ en muestras de agua fue $0,007,0,007$ y $0,01 \mathrm{mg} / \mathrm{L}$ para las zonas A, B y C respectivamente. En el mismo orden, la concentración de $\mathrm{Hg}$ fue $0,063,0,032$ y $0,171 \mathrm{mg} / \mathrm{L}$. y de $\mathrm{Cd} 0,000 \mathrm{mg} / \mathrm{L}$ para las tres zonas, respectivamente. En las tres zonas el $\mathrm{Hg}$ estuvo por encima de los niveles permitidos por la Agencia de Protección del Medio Ambiente de los Estados Unidos. Se concluye que peces y agua representan potenciales medios de contaminación de personas con metales pesados a partir de la ruta digestiva en esta zona.

PALABRAS CLAVE: metales pesados, plomo, mercurio, cadmio, peces, Huaraz

\section{SUMMARY}

The objective of the study was to quantifies the concentration of $\mathrm{Pb}, \mathrm{Hg}$ and $\mathrm{Cd}$ in muscle of fish destined for human consumption and in samples of river water in three locations ( $\mathrm{A}=\mathrm{Catac}, \mathrm{B}=\mathrm{C}=$ Taricá and Palmira) from the city of Huaraz (Ancash, Peru). Five fish and water samples at each site were analyzed by the Flame Atomic Absorption technique. The concentration of $\mathrm{Pb}$ in fish muscle was $1.826,0.321$ and $0.709 \mathrm{mg} / \mathrm{kg}$ for zones A, B and $\mathrm{C}$ respectively. In the same order, the concentration of $\mathrm{Hg}$ was $0.344,0.433$ and $0.284 \mathrm{mg} / \mathrm{kg}$ and $\mathrm{Cd}, 0.001$, 0.0 and $0.002 \mathrm{mg} / \mathrm{kg}$ respectively. In all three areas, the concentrations of $\mathrm{Pb}$ were above the levels permitted by the European Regulatory Commission. The concentration of $\mathrm{Pb}$ in water samples was $0.007,0.007$ and $0.01 \mathrm{mg} / \mathrm{L}$ for zones A, B and C respectively. In the same order, the concentration of $\mathrm{Hg}$ was $0.063,0.032$ and $0.171 \mathrm{mg} / \mathrm{L}$. $\mathrm{Cd}$ and $0.000 \mathrm{mg} / \mathrm{L}$ for the three areas respectively. In all three areas the $\mathrm{Hg}$ was above the levels permitted by the Agency for Environmental Protection of the United States. It is concluded that fish and water represent potential media for contamination of persons with heavy metals via the digestive tract, in this area.

KEYWORDS: heavy metals, lead, mercury, cadmium, fish, Huaraz 


\section{INTRODUCCIÓN}

La presencia de metales pesados en los ecosistemas constituye un serio problema debido a su elevada persistencia medioambiental y su alta toxicidad para los organismos vivos; ejemplo de ellos son el cadmio $(\mathrm{Cd})$, plomo $(\mathrm{Pb})$ y mercurio $(\mathrm{Hg})$ (Kolf-Clauw, Guénin y, López, 2007). Estos tienen la propiedad de ser bioacumulables, pues al organismo afectado le resulta imposible mantener la velocidad necesaria de excreción (Mendoza, Soler y Pérez, 2008).

Los metales pueden ser absorbidos por difusión pasiva, transporte activo o endocitosis (Veltman, Huijbregts, Kolck, Wang y Hendriks, 2008) y la biodisponibilidad de ellos, va a depender de su forma química (Jacinto y Aguilar, 2007).

Elcadmiopuedeafectarelriñónproduciendocuadros de insuficiencia renal, también puede producir lesión hepática, convulsiones, shock e inclusive puede afectar huesos y sangre en caso de exposiciones prolongadas (García y Cruz, 2012). El mercurio se acumula en corrientes fluviales y océanos, convirtiéndose en mercurio metílico (Environmental Protection Agency (EPA) y Food and Drug Administration (FDA), 2004). Los peces son la fuente dietética de mercurio metílico potencialmente neurotóxicos para los seres (Hightower y Moore, 2003). La extracción de oro artesanal es la principal fuente de contaminación principalmente como mercurio metálico (Ministerio de Energía y Minas (MINEM), 2005; Boudou, Maury-Brachet, Coquery, Durrieu y Cossa, 2005). El plomo proviene generalmente del polvo contaminado y del agua potable. Atraviesa la placenta y penetra en el cerebro fetal, interfiriendo su desarrollo normal. Puede llegar a producir problemas en la síntesis de hemoglobina, efecto sobre los riñones, del aparato gastrointestinal, sistema reproductivo y daños al sistema nervioso (Lenntech (s.f.)).

Los moluscos, como organismos filtradores, y los peces tienden a acumular metales en sus tejidos (Burger y Gochfeld, 2005). Para cada elemento traza, los sitios de acumulación en los peces puede variar según la vía de absorción y, también con la intensidad y duración de la exposición (Ciardullo et al., 2008).

La Comisión Reguladora Europea (EC) No 1881/2006 pone límites máximos para algunos contaminantes en los alimentos, entre ellos $\mathrm{Pb}, \mathrm{Hg}$ y Cd. (Comission of the European Communities (EC), 2008). Se ha establecido límites máximo permisibles para consumo humano en filetes de pescado (músculo de peces) (EC, 2008) y para el agua (EPA, 2009).

En este contexto el estudio tuvo por objetivo cuantificar la concentración de $\mathrm{Pb}, \mathrm{Hg}$ y Cd en músculo de peces comercializados para consumo humano y en muestras de agua procedentes del Río Santa (Ancash Perú) a los que tienen acceso los pobladores de la zona para alimentación o bebida.

\section{MATERIAL Y MÉTODOS}

El estudio se realizó en la ciudad de Huaraz, departamento de Ancash, ubicado en la zona noroccidental del Perú, con una extensión de 35 826 kilómetros cuadrados y su población es de 1 014163 habitantes (Instituto Nacional de Estadística e Informática (INEI), 1996). La ciudad se encuentra situada sobre los $3100 \mathrm{msnm}$ y a $408 \mathrm{Km}$. del departamento de Lima.

El estudio transversal descriptivo consideró el muestreo de peces y agua. Se tomaron muestras en tres puntos diferentes de comercialización de peces, lo que correspondió a un total de 15 muestras de peces. Los peces utilizados fueron truchas Arco Iris (Oncorhynchus mykiss) de género indefinido, pesando entre $20 \mathrm{gr}-220$ gr y midiendo entre $13 \mathrm{~cm}-29 \mathrm{~cm}$. Además, en cada una de las zonas se tomó una muestra de agua directamente del Río Santa.

Las zonas de muestreo fueron las siguientes:

- Zona A.- correspondió al poblado de Shiki - Catac, ubicado a $3640 \mathrm{msnm}$ y a $48 \mathrm{~km}$ hacia el Sur de Huaraz.

- Zona B.- correspondió al centro de la ciudad, en la urbanización de Palmira, ubicándose a $4 \mathrm{~km}$ del mismo.

- Zona C.- correspondió al centro poblado Paltay Taricá, ubicado a $20 \mathrm{~km}$ hacia el Norte de Huaraz.

Las muestras de peces se conservaron refrigeradas en un envase hermético a temperatura de refrigeración $\left(4\right.$ a $8{ }^{\circ} \mathrm{C}$ ) y fueron trasladados a la ciudad de Lima dentro de las primeras 24 horas de obtenidos. Las muestras de agua fueron recolectadas directamente del río Santa y se almacenaron en botellas de plástico de un litro de capacidad. Estas fueron selladas y refrigeradas inmediatamente después de la recolección para ser enviadas al laboratorio de diagnóstico.

El análisis fue realizado por la Unidad de Servicios de Análisis Químicos (USAQ) de la Universidad 
Nacional Mayor de San Marcos, mediante la técnica de absorción atómica de flama (AASF).

Los resultados fueron resumidos mediante estadísticas descriptivas. Se comparó la concentración de cada uno de los metales pesados encontrados en las muestras de peces y agua con los valores mínimos permisibles por la norma de la Unión Europea. La diferencia entre las medias de concentración de $\mathrm{Hg}, \mathrm{Pb}$ y $\mathrm{Cd}$ en los puntos de recolección de las muestras se evaluó mediante la prueba de Análisis de Varianza de una vía y la prueba de Diferencia de Medias de Tukey.

\section{RESULTADOS}

Durante el mes de febrero del año 2011 se recolectaron 15 muestras de peces que se comercializaban en tres zonas diferentes de la ciudad de Huaraz. Cinco peces fueron obtenidas en la zona de Catac, inmediatamente después de la pesca artesanal en río y, 10 muestras en las zonas de Palmira y Taricá en donde se obtuvieron peces procedentes de lagunas.

Según el lugar en los que los animales fueron criados (río o laguna) se encontró diferencia significativa en el tamaño y peso, siendo menor en los peces procedentes de la zona de Catac (tabla 1).

Se observó diferencia significativa en la concentración de plomo en los peces obtenidos en la zona de Catac y Palmira $(\mathrm{p}<0.05)$, siendo mayor en Catac. No se observó diferencia para Mercurio, pero si para Cadmio. En este último caso, Catac y Taricá tuvieron una concentración mayor en comparación a Palmira $(\mathrm{p}<0,05)$ (tabla 2). Las concentraciones de $\mathrm{Pb}$ fueron mayores que las permisibles para peces por la EC en las 3 zonas. En caso del $\mathrm{Hg}$, los niveles estuvieron por encima de los permisibles por la EC en la zona de Palmira.

Tabla1. Características del lugar y muestras de peces obtenidas para el estudio de metales pesados en diferentes zonas del Departamento de Ancash - Perú, 2011.

\begin{tabular}{|c|c|c|c|c|c|}
\hline \multirow{2}{*}{$\begin{array}{l}\text { Lugar de } \\
\text { crianza }\end{array}$} & \multirow[t]{2}{*}{ Zona de Muestreo } & \multirow{2}{*}{$\begin{array}{l}\text { Ubicación respecto a } \\
\text { la ciudad }\end{array}$} & \multirow{2}{*}{$\begin{array}{l}\text { Muestras } \\
\text { Obtenidas }\end{array}$} & \multicolumn{2}{|c|}{$\begin{array}{l}\text { Dimensiones promedio de las } \\
\text { muestras }\end{array}$} \\
\hline & & & & Peso (gr.) & Talla (cm.) \\
\hline Río & Catac & $48 \mathrm{Km}$ al Sur & 5 & $20^{\mathrm{a}}$ & $14,2^{\mathrm{a}}$ \\
\hline \multirow{2}{*}{ Laguna } & Palmira & $4 \mathrm{Km}$ al Este & 5 & $208^{b}$ & $25,4^{b}$ \\
\hline & Taricá & $20 \mathrm{Km}$ al Norte & 5 & $204^{b}$ & $24,8^{b}$ \\
\hline
\end{tabular}

a,b Letras diferentes en las medias indican que los valores encontrados son significativamente diferentes para cada variable

Tabla 2. Resultados de la evaluación de metales pesados en peces obtenidos y comercializados en diferentes zonas del Departamento de Ancash - Perú, 2011.

\begin{tabular}{|c|c|c|c|c|c|}
\hline \multirow{2}{*}{$\begin{array}{c}\text { Metal } \\
\text { estudiado }\end{array}$} & \multirow{2}{*}{ Zona de Muestreo } & \multirow{2}{*}{ Media } & \multirow{2}{*}{$\begin{array}{c}\text { Desviación } \\
\text { Estándar }\end{array}$} & \multicolumn{2}{|c|}{ Valores Extremos } \\
\hline & & & & Mínimo & Máximo \\
\hline \multirow{3}{*}{$\begin{array}{l}\text { Plomo } \\
(\mathrm{mg} / \mathrm{Kg})\end{array}$} & Catac & $1,826^{\mathrm{a}}$ & 1,299 & 0,002 & 2,920 \\
\hline & Palmira & $0,322^{\mathrm{b}}$ & 0,429 & 0,000 & 0,873 \\
\hline & Taricá & $0,710^{\mathrm{ab}}$ & 0,595 & 0,000 & 1,379 \\
\hline \multirow{3}{*}{$\begin{array}{l}\text { Mercurio } \\
(\mathrm{mg} / \mathrm{Kg})\end{array}$} & Catac & $0,344^{\mathrm{a}}$ & 0,118 & 0,218 & 0,471 \\
\hline & Palmira & $0,434^{\mathrm{a}}$ & 0,313 & 0,238 & 0,988 \\
\hline & Taricá & $0,284^{\mathrm{a}}$ & 0,186 & 0,132 & 0,598 \\
\hline \multirow{3}{*}{$\begin{array}{l}\text { Cadmio } \\
(\mathrm{mg} / \mathrm{Kg})\end{array}$} & Catac & $0,001^{\mathrm{a}}$ & 0,001 & 0,000 & 0,002 \\
\hline & Palmira & $0,000^{\mathrm{b}}$ & 0,000 & 0,000 & 0,000 \\
\hline & Taricá & $0,002^{\mathrm{a}}$ & 0,001 & 0,001 & 0,003 \\
\hline
\end{tabular}

${ }^{\mathrm{a}, \mathrm{b}}$ Letras diferentes en las medias indican que los valores encontrados son significativamente diferentes 
Tabla 3. Resultados de la evaluación de metales pesados en peces distribuidos según procedencia de crianza en rio $(n=5)$ o Laguna $(n=10)$, en el Departamento de Ancash - Perú, 2011.

\begin{tabular}{|c|c|c|c|c|c|}
\hline \multirow{2}{*}{$\begin{array}{c}\text { Metal } \\
\text { estudiado }\end{array}$} & \multirow{2}{*}{$\begin{array}{l}\text { Procedencia de la } \\
\text { Muestra }\end{array}$} & \multirow{2}{*}{ Media } & \multirow{2}{*}{ Desviación Estándar } & \multicolumn{2}{|c|}{ Valores Extremos } \\
\hline & & & & Mínimo & Máximo \\
\hline \multirow{2}{*}{$\begin{array}{l}\text { Plomo } \\
(\mathrm{mg} / \mathrm{Kg})\end{array}$} & Río & $1,826^{\mathrm{a}}$ & 1,299 & 0,002 & 2,920 \\
\hline & Laguna & $0,516^{\mathrm{b}}$ & 0,530 & 0,0 & 1,379 \\
\hline \multirow{2}{*}{$\begin{array}{l}\text { Mercurio } \\
(\mathrm{mg} / \mathrm{Kg})\end{array}$} & Río & $0,344^{\mathrm{a}}$ & 0,118 & 0,218 & 0,471 \\
\hline & Laguna & $0,359^{\mathrm{a}}$ & 0,255 & 0,132 & 0,988 \\
\hline \multirow{2}{*}{$\begin{array}{l}\text { Cadmio } \\
(\mathrm{mg} / \mathrm{Kg})\end{array}$} & Río & $0,001^{\mathrm{a}}$ & 0,000 & 0,000 & 0,001 \\
\hline & Laguna & $0,000^{\mathrm{a}}$ & 0,000 & 0,000 & 0,003 \\
\hline
\end{tabular}

${ }^{a, b}$ Letras diferentes en las medias indican que los valores encontrados son significativamente diferentes $(p<0.05)$

Tabla 4. Resultados de la evaluación de metales pesados en muestras de agua procedente de diferentes zonas del río Santa en el Departamento de Ancash - Perú, 2011.

\begin{tabular}{lccc}
\hline Lugar de muestreo & $\begin{array}{c}\text { Plomo } \\
(\mathrm{mg} / \mathrm{L})\end{array}$ & $\begin{array}{c}\text { Mercurio } \\
(\mathrm{mg} / \mathrm{L})\end{array}$ & $\begin{array}{c}\text { Cadmio } \\
(\mathrm{mg} / \mathrm{L})\end{array}$ \\
\hline Catac & 0,007 & 0,063 & 0,000 \\
Palmira & 0,007 & 0,032 & 0,000 \\
Taricá & 0,010 & 0,171 & 0,000 \\
\hline
\end{tabular}

Se encontró una mayor concentración de plomo $(\mathrm{p}<0,05)$ en peces procedentes del Río Santa en comparación a los de laguna (tabla 3). No se encontró diferencia en la concentración de $\mathrm{Hg}$ y $\mathrm{Cd}$. Cuando se compara la concentración de $\mathrm{Pb}$ según lugar de procedencia de los peces, se encuentra que estas están por encima de lo permitido por la EC, tanto en laguna como en río.

No se encontraron concentraciones de Plomo mayores a las permisibles por la EPA en ninguna de las 3 zonas muestreadas. En el caso del Mercurio sucedió lo contrario, se encontraron concentraciones mayores que las permisibles por la EPA en las 3 zonas, siendo mayor la concentración de este metal para la zona de Taricá. La concentración del Cadmio sin embargo, no se encontró elevada en ninguna zona (tabla 4).

\section{DISCUSIÓN}

En las localidades estudiadas de Huaraz, la mayoría de personas compran sus pescados en mercados que se encuentren cerca al lugar donde viven o lo adquieren a pescadores artesanales en dichas zonas. Los peces que se venden pueden tener dos orígenes, provienen de laguna o del río Santa. Lo que no se conoce es si estos animales acumulan en su organismo algún tipo de contaminante como metales pesados. Gale, Adams, Wixson, Loftin y Huang (2002) mencionan que el músculo de peces es la porción del animal que típicamente se consume, por ello el estudio evaluó la concentración de metales pesados en la musculatura.

Los peces de mayor tamaño y peso fueron los de las zonas de Palmira y Taricá, que correspondían a peces procedentes de piscigranjas; mientras que los de menor peso y tamaño fueron los de la zona de Catac que correspondieron a peces extraídos del rio.

La presencia de $\mathrm{Pb}$ en la musculatura de peces fue mayor en aquellos que provenían de río (Catac). Sin embargo, en todas las zonas, los niveles de $\mathrm{Pb}$ encontrados en la musculatura de los peces, estuvieron por encima del límite máximo permisible por la $\mathrm{EC}$, con lo que se puede inferir que existe contaminación por este metal en los ambientes en los que se crían estos peces. En un estudio realizado por Gale et al., (2002) en EEUU, encontraron concentraciones elevadas de $\mathrm{Pb}$ en sedimentos de río y peces, debido a la erosión del suelo a causa del escurrimiento de los depósitos de mineral en la superficie y presencia de relaves en las actividades mineras. 
Además, Kolf-Claw et al., (2007), mencionan que la contaminación por $\mathrm{Pb}$ puede darse por diferentes vías, ya sea natural como el vulcanismo y la erosión eólica, o antropogénica como la polución automovilística y la industrial como es el caso de las actividades metalúrgicas, importante en el departamento de Ancash.

En el caso del $\mathrm{Hg}$, los resultados estuvieron por debajo de los límites máximos permisibles en músculo de peces establecidos por la EC; esto se puede deber a una distribución natural de los metales pesados en el organismo. Ciardullo et al., (2008) mencionan que las concentración de diversos metales incluyendo el $\mathrm{Hg}$ se encuentran en menor cantidad en piel y musculo de peces, en comparación a otros órganos (branquias, riñón, hígado). Asimismo, De bruyn y Gobas (2006) mencionan que el grado en que se acumulan los contaminantes alimentarios varía mucho entre especies pudiéndose atribuir en gran parte a la variación en la forma como las especies se alimentan, digieren y asignan su energía.

$\mathrm{La}$ fuente principal de contaminación por $\mathrm{Hg}$ estaría en la minería artesanal. El MINEM (2005), menciona que se utiliza el $\mathrm{Hg}$ para la recuperación del oro debido a la facilidad que tiene para formar compuestos (amalgamas) con los metales preciosos. Se sabe además que, las plantas de amalgamación se encuentran ubicadas generalmente a orillas de los cursos de agua o cerca de ellos. Por ello, la contaminación de las aguas por $\mathrm{Hg}$ haría evidente la presencia de minas artesanales cercanas al río o lagunas.

Boudou et al., (2005) mencionan que los peces depredadores y de mayor tamaño, tenían la mayor concentración de $\mathrm{Hg}$ en su organismo debido a que los peces piscívoros como es el caso de la trucha, tienden a acumular más metales en su organismo. Oscoz, Escala y Campos (2000) afirma que un pez adulto se alimenta con otro tipo de organismos, confirmando la capacidad de los peces para acumular y biomagnificar los metales pesados. Estos factores no podrían atribuirse necesariamente a los animales del estudio, sin embargo la presencia de metales pesados sugiere la presencia de contaminación en su hábitat.

En el caso del $\mathrm{Cd}$, los resultados para las zonas de Catac y Taricá fueron los mayores, sin superar los límites máximo permisibles. Para la zona de Palmira, no se detectó Cd. El MINEM (2005) menciona que Cd se obtiene principalmente en los procesos de refinación del zinc y en menor grado en la refinación de plomo, concentrados de zinc y cobre.

El límite máximo permisible para músculo de peces en el caso del $\mathrm{Cd}$ es de $0,050 \mathrm{mg} / \mathrm{Kg}$. Eissa, Salibian, Ferrari y Pérez (2005) señalan que el Cd es un elemento que en muy bajas cantidades afecta mecanismos y funciones fundamentales de diferentes especies, incluidos los humanos, siendo los órganos blanco más importantes el hígado y el riñón, por lo que las organizaciones tales como la $\mathrm{EC}$, ponen límites máximos permisibles muy bajos.

La diferencia en los niveles de metales pesados en la musculatura de peces con respecto a procedencia y lugar de muestreo, se pueden explicarpor las variaciones geográficas, niveles tróficos, tamaño de los peces y la capacidad de cada metal para biomagnificarse en la cadena alimenticia. Burger y Gochfeld (2005) afirman que es de esperar que los peces que están más arriba en la cadena trófica acumulen niveles más altos de metales tales como el Hg en su organismo. Asimismo, Boyle et al., (2008) mencionan que algunos metales se acumulan dependiendo del tamaño y edad del pez. Es decir, los invertebrados acuáticos que viven en ambientes contaminados por metales pesados pueden contenerlos también en su organismo, y así contribuir potencialmente a la acumulación, magnificación y cronicidad de estos elementos en especies predadoras, tal como es el caso de los peces.

Respecto a los resultados de las muestras de agua, estas mostraron que sólo en el caso del $\mathrm{Hg}$ se encontraron concentraciones mayores a las permisibles por la EPA en las tres zonas de estudio, ello podría explicarse por un fenómeno acumulativo por arrastre de contaminantes. Escobar (2003) menciona que alrededor del $70-75 \%$ de la contaminación marina global es producto de las actividades humanas que tienen lugar en la superficie terrestre y un $90 \%$ de los contaminantes es transportado por los ríos al mar, por ello la importancia de monitorear las aguas de río periódicamente.

Siguiendo las apreciaciones de Gale et al., (2002), resultaría apropiado analizar muestras de sedimentos en el río, debido a que existe la hipótesis de que la mayoría del $\mathrm{Pb}$ encontrado en muestras de peces se debe a la presencia de este metal en partículas orgánicas presentes en el sedimento e incluso en peces contaminados que luego son ingeridos por otros. 
Se espera que los resultados de la presente investigación permitan dilucidar el rol de los peces y el agua como potenciales vectores de contaminación con metales pesados de las personas, a partir de la ruta digestiva, y poner en alerta del mismo a las autoridades a fin de implementar medidas de prevención $\mathrm{y}$ protección de las poblaciones humanas adyacentes al río Santa.

\section{CONCLUSIONES}

El estudio llegó a las siguientes conclusiones:

- Las concentraciones de $\mathrm{Pb}$ fueron mayores que las permisibles por la EC para músculo de peces en las tres zonas (Catac, Taricá y Palmira). Así como el lugar de procedencia (laguna y rio). En caso del $\mathrm{Hg}$, los niveles estuvieron por encima de los permisibles en la zona de Palmira.

- Se encontró concentraciones de Hg mayores a las permisibles por la EPA en muestras de agua en las tres zonas muestreadas. No hubo diferencia para el caso de $\mathrm{Pb}$ y $\mathrm{Cd}$.

\section{Correspondencia:}

Flavia Bertolotti Rivera

Correo electrónico: flavia.bertolotti@gmail.com

\section{REFERENCIAS BIBLIOGRÁFICAS}

1. Boudou, A., Maury-Brachet, R., Coquery, M., Durrieu, G., \& Cossa, D. (2005). Synergic effect of gold mining and damming on mercury contamination in fish. Environmental Science \& Technology, 39(8), 2448-2454.

2. Boyle, D., Brix, K. V., Amlund, H., Lundebye, A. K., Hogstrand, C., \& Bury, N. R. (2008). Natural arsenic contaminated diets perturb reproduction in fish. Environmental science \& technology, 42(14), 5354-5360.

3. Burger, J., \& Gochfeld, M. (2005). Heavy metals in commercial fish in New Jersey. Environmental Research, 99(3), 403-412.

4. Ciardullo, S., Aureli, F., Coni, E., Guandalini, E., Iosi, F., Raggi, A., ... \& Cubadda, F. (2008). Bioaccumulation potential of dietary arsenic, cadmium, lead, mercury, and selenium in organs and tissues of rainbow trout (Oncorhyncus mykiss) as a function of fish growth. Journal of agricultural and food chemistry, 56(7), 2442-2451.

5. De Bruyn, A. M., \& Gobas, F. A. (2006). A bioenergetic biomagnification model for the animal kingdom. Environmental science \& technology, 40(5), 1581-1587.

6. Comission of the European Communities. Comission Regulation No 629/2008. Ammending Regulation (EC) No 1881/2006. Setting maximum levels for certain contaminants in foodstuffs. Official Journal of the European Union, 173, 6-9.

7. Eissa, B. L., Salibian, A., Ferrari, L., \& Pérez, R. (2005). Estudios sobre los efectos etológicos del Cadmio. Roma: Organización de las Naciones Unidas para la alimentación y agricultura. Recuperado de: http://agris.fao.org/agris-search/ search.do?recordID=AV20120131168

8. Environmental Protection Agency. (2009). National primary drinking water regulations. Washington: Environmental Protection Agency.

9. Environmental Protection Agency and Food and Drug Administration. (2004). Lo que usted necesita saber sobre el mercurio en el pescado y los mariscos. Washington: Environmental Protection Agency. Recuperado de: $\quad$ https://www.epa.gov/choose-fish-andshellfish-wisely/lo-que-usted-necesita-sabersobre-el-mercurio-en-el-pescado-y-los

10. Escobar, J. (2003). La contaminación de los ríos y sus efectos en las áreas costeras y el mar. Washington: United Nations Publications.

11. Gale, N. L., Adams, C. D., Wixson, B. G., Loftin, K. A., \& Huang, Y. W. (2002). Lead concentrations in fish and river sediments in the old lead belt of Missouri. Environmental science \& technology, 36(20), 4262-4268.

12. García, J. O., Tortajada, J. F., Conesa, A. C., \& Castell, J. G. (2005). Neurotóxicos medioambientales (y II). Metales: efectos adversos en el sistema nervioso fetal y posnatal. Acta Pediatr Esp, 63, 182-192.

13. García, P. E. P., \& Cruz, M. I. A. (2012). Los efectos del cadmio en la salud. Revista de Especialidades Médico-Quirúrgicas, 17(3), 199-205.

14. Instituto Nacional de Estadística e Informática. (1996). Perú: Población total tasa de crecimiento $e$ indice de masculinidad según departamento, 1995-2000. Lima: Instituto Nacional de Estadística e Informática.

15. Mendoza García, M., Soler Rodríguez, F., \& Pérez López, M. (2008). Los mamíferos salvajes terrestres como bioindicadores: nuevos avances en Ecotoxicología. Unidad de Toxicología. Facultad de Veterinaria (UEX). Universidad de Extremadura.

Observatorio Medioambiental, 37(11), 37-62.

16. Hightower, J. M., \& Moore, D. (2003). Mercury levels in high-end consumers of fish. Environmental 
health perspectives, 111(4), 604.

17. Jacinto, M. E., \& Aguilar, S. (2007). Concentraciones traza de metales en especies marinas de la bahía de Huarmey, Ancash, Perú. Revista Peruana de Biología, 14(2), 307312.

18. Kolf-Clauw, M., Guénin, A., \& López, M. P. (2007). Micromamíferos y metales pesados: Biomonitorización del medio ambiente/ Micromammals and heavy metals: Environmental Biomonitoring/Micromammifèresetmetaux lourds: Biomonitoring de l'environnement. Observatorio Medioambiental, 10, 19.
19. Ministerio de Energía y Minas, Dirección general de minería. (2005). Guía Minera 2005. Uso y Manejo del Mercurio. Lima: Ministerio de Energía y Minas. Disponible en: http://www.minem.gob. $\mathrm{pe} / \mathrm{minem} / \mathrm{archivos} / \mathrm{file} / \mathrm{Mineria/}$ PUBLICACIONES/GUIAS/GUIAS/UMM\%20 2004.pdf

20. Oscoz, J., Escala, M. C., \& Campos, F. (2000). La alimentación de la trucha común (Salmo trutta L., 1758) en un río de Navarra (N. España). Limnetica, 18(1), 29-35.

21. Veltman, K., Huijbregts, M. A., Kolck, M. V., Wang, W. X., \& Hendriks, A. J. (2008). Metal bioaccumulation in aquatic species: quantification of uptake and elimination rate constants using physicochemical properties of metals and physiological characteristics of species. Environmental science \& technology, 42(3), 852-858. 